\title{
The Role of Feedback in Shaping the Abundance Gradients and Age-Metallicity Relations of Galaxies
}

\author{
Brad K. Gibson,, ${ }^{* a}$ Kate Pilkington, ${ }^{a}$, \\ Jeremy Bailin, ${ }^{b}$, Chris B. Brook, ${ }^{c}$ and Greg S. Stinson ${ }^{d}$ \\ a Jeremiah Horrocks Institute, University of Central Lancashire \\ ${ }^{b}$ Dept of Physics \& Astronomy, University of Alabama \\ ${ }^{c}$ Dept de Física Teórica, Universidad Autónoma de Madrid \\ ${ }^{d}$ Max-Planck-Institut für Astronomie, Heidelberg \\ E-mail: brad.k.gibson@gmail.com
}

\begin{abstract}
We examine the role of energy feedback in shaping the distribution of metals within cosmological hydrodynamical simulations of $\mathrm{L}^{*}$ disk galaxies. While negative abundance gradients today provide a boundary condition for galaxy evolution models, in support of inside-out disk growth, empirical evidence as to whether abundance gradients steepen or flatten with time remains highly contradictory. Making use of a suite of $\mathrm{L}^{*}$ disks, realised with and without 'enhanced' feedback, we show that relatively 'flat' and temporally invariant abundance gradients are a natural outcome of feedback schemes which distribute energy and re-cycled ISM material over large scales. Our 'enhanced' feedback models, which have been shown to be in remarkable agreement with a broad range of empirical scaling relations, predict that gradients should steepen mildly with time. Such a conclusion is consistent with extant analytical models of galactic chemical evolution, and inconsistent with our earlier generation of simulations realised with 'conventional' feedback schemes. Enhanced feedback results in significantly reduced scatter in the local stellar age-metallicity relation and, especially, the $[\mathrm{O} / \mathrm{Fe}]-[\mathrm{Fe} / \mathrm{H}]$ distribution. The local $[\mathrm{O} / \mathrm{Fe}]$ distribution function for our $\mathrm{L}^{*}$ disks show clear bimodality, with peaks at $[\mathrm{O} / \mathrm{Fe}]=-0.05$ and +0.05 (for stars with $[\mathrm{Fe} / \mathrm{H}]>-1$ ), consistent with our earlier work on dwarf disks and observations of the local solar neighbourhood of the Milky Way.
\end{abstract}

XII International Symposium on Nuclei in the Cosmos

August 5-12, 2012

Cairns, Australia

${ }^{*}$ Speaker. 


\section{Introduction}

Radial abundance gradients and stellar age-metallicity relations provide powerful constraints on the complex interplay between gas infall (e.g., cold flows from the IGM, coronal re-cycling of the underlying ISM), outflows (e.g., galactic fountains, superwinds, mass loading), stellar migration and radial gas flows, secular kinematic meeting, interaction- and merger-driven energetics, and star formation efficiency, in driving the 'inside-out' growth of disk galaxies. Local disks, including the Milky Way, provide one critical 'boundary condition' for all models, in the sense that their present-day (i.e., gas-phase) metallicity gradients must be 'negative' (i.e., decreasing in metallicity with increasing galactocentric radius) and of the order $-0.04 \mathrm{dex} / \mathrm{kpc} .{ }^{1}$ Observationally though, there has been little or no associated boundary conditions on the temporal evolution of said metallicity gradients. This has changed with the first in situ determinations of radial abundance gradients in typical star forming spirals at redshifts $2.4<z<1.0[8,15,24]$.

Motivated by these unique empirical constraints, we examine here the role of energy feedback in shaping the temporal evolution of abundance gradients and age-metallicity relations within a subset of cosmological hydrodynamical disk simulations drawn from the MUGS [21] and MaGICC [3] suites. We will demonstrate how such observations can genuinely constrain the highly uncertain nature and magnitude of energy feedback underpinning galaxy formation.

\section{Simulations}

We make use of two simulations (g1536; g15784) drawn from the MUGS [21] suite of L* cosmological disks. Realised with the SPH code Gasoline, two variants of each were analysed - one using 'conventional' feedback (MUGS) and one using 'enhanced' feedback (MaGICC). Full details, including the methodology associated with star formation and feedback, for MUGS, can be found in Stinson et al. [21] and Pilkington et al. [12]. The MaGICC feedback prescription is outlined in a series of papers by Brook et al., Stinson et al., and Pilkington et al. [2-4, 14, 22, 23].

Briefly, MUGS-g1536 and MUGS-g15784 employ a thermal feedback scheme in which $4 \times 10^{50}$ erg per supernova (SN) is made available to heat the surrounding ISM, while their MaGICC analogs make use of $10^{51} \mathrm{erg} / \mathrm{SN}$. The MUGS (MaGICC) simulations were realised with a Kroupa et al. [9] (Chabrier: [5]) IMF. ${ }^{2}$ For the MaGICC runs, radiation energy feedback from massive stars is also included (in the $\sim 4$ Myr prior to a star particle's formation and the appearance of the first Type II SN from said particle), albeit at an effective coupling efficiency $<1 \%[3,23]$. For both MUGS and MaGICC, cooling is disabled for gas particles situated within a blast region of size $\sim 100 \mathrm{pc}$, for a time period of $\sim 10$ Myr. Star formation is restricted to regions which are both sufficiently cool $(<10 \mathrm{kK})$ and dense (MUGS: $>1 \mathrm{~cm}^{-3}$; MaGICC: $\left.>9 \mathrm{~cm}^{-3}\right)$.

To link the simulation nomenclature employed here with their earlier appearances in the literature, MUGS-g1536 and MUGS-g15784 correspond to g1536 and g15784, respectively [12,21], while MaGICC-g1536 and MaGICC-g15784 correspond to 'Fiducial' [23] and the g15784 ana$\log$ to the 'Low Diffusion' realisation of Stinson et al. [23], respectively. ${ }^{3}$

\footnotetext{
${ }^{1}$ At least within the actively star-forming parts of the disks (within $\sim 3-5$ disk scalelengths); gradients in the outer disk can be negative, flat, or even inverted (e.g. Spitoni \& Matteucci 2011, and references therein).

${ }^{2}$ The MUGS runs assumed that the global metallicity $\mathrm{Z} \equiv \mathrm{O}+\mathrm{Fe}$, while those of MaGICC assume $\mathrm{Z} \equiv \mathrm{O}+\mathrm{Fe}+\mathrm{C}+\mathrm{N}+\mathrm{Ne}+\mathrm{Mg}+\mathrm{Si}$; as such, the MUGS simulations underestimate the global metallicity by roughly a factor of two, and hence the impact of metallicity-dependent cooling [12].

${ }^{3}$ MaGICC-g1536 is also essentially the same as SG5LR, as reported in our earlier work [3].
} 


\section{Abundance Gradients}

In Pilkington et al. [12], we showed that 'conventional' feedback schemes (i.e., those making use of $\sim 10-40 \%$ of the energy associated with each SN, to heat the surrounding ISM), when coupled with a classical SPH approach to hydrodynamics (independent of the SPH code employed), tended to result in galaxies with steep abundance gradients at redshifts $z>1(-0.15 \rightarrow-0.30 \mathrm{dex} / \mathrm{kpc}$ vs $-0.04 \mathrm{dex} / \mathrm{kpc}$, today). Such 'conventional' feedback schemes, when coupled to a grid-based approach to hydrodynamics at roughly the same resolution, also led to gradients steeper at highredshift relative to the predicted present-day values $(-0.05 \rightarrow-0.10 \mathrm{dex} / \mathrm{kpc}$ vs $-0.04 \mathrm{dex} / \mathrm{kpc}$, today), although systematically shallower than their SPH counterparts. This apparent systematic difference was driven only by the particular grid-based approach being adopted imposing a twogrid-cell minimum to the blastwave radius. We concluded then that any feedback scheme which distributed energy more efficiently on larger scales should result in flatter gradients.

At the time of this earlier work [12,13], we did not have a clear manner in which to quantify the above inference. We are now in a position to show, in a direct manner, the impact on the temporal evolution of abundance gradients for $\mathrm{L}^{*}$ disk galaxies, when replacing the conventional feedback scheme employed for MUGS-g1536 and MUGS-g15785 with the well-tested MaGICC scheme.

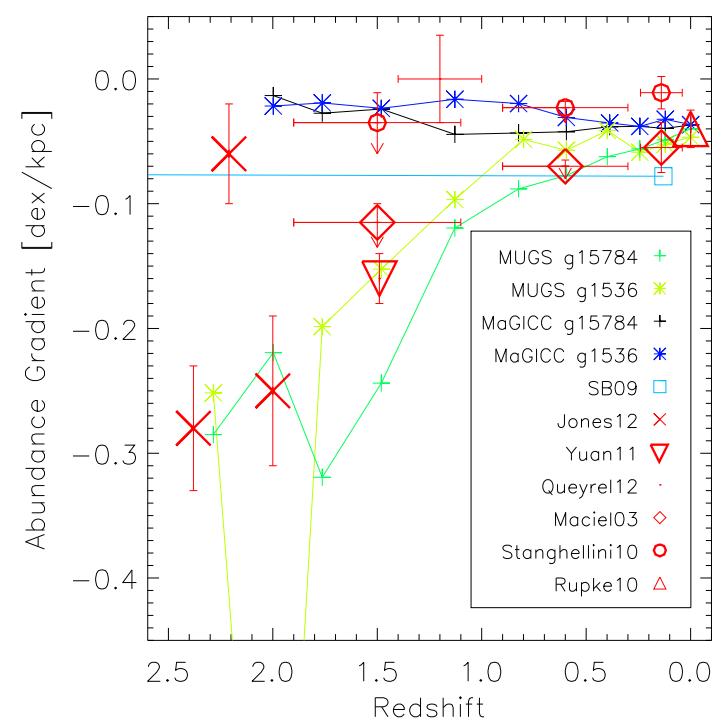

Figure 1: Temporal evolution of the predicted oxygen abundance gradients associated with four simulated $\mathrm{L}^{*}$ disk galaxies - from the MUGS [21] and MaGICC [3] suites - in addition to an analytical model (SB09: [18]). Symbols correspond to empirical determinations of the abundance gradients in a sample of high-redshift lensed systems (Yuan11: [24]; Jones12: [8]), intermediate-redshift galaxies from MASSIV (Queyrel12: [15]), local disks (Rupke10: [16]), and the Milky Way (Maciel03: [10]; Stanghellini10: [20]).

In Fig 1, we show the evolution of the gas-phase oxygen abundance gradients for both sets of realisations (g15784: + symbols; g1536: * symbols); the lower ('conventional' feedback) pair show the significant flattening from $z \sim 2$ to $z \sim 0$ first described by Pilkington et al. [12]. Conversely, the upper pair show a dramatically different evolutionary sequence; the stronger feedback imple- 
mented within the MaGICC scheme, results in essentially flat gradients at high-redshift, with minimal steepening (rather than flattening) with time. As the MaGICC feedback scheme re-distributes energy and re-cycled ISM material over much greater galactic scales, such flat (and temporallyindependent) gradients is consistent with our interpretation of the difference between the grid and particle-based simulations, as noted above, and in our earlier work [12].

Prior to 2011, no in situ measurements of abundance gradients at $z>0$ existed (particularly for 'typical' star-forming and/or Grand Design spirals). Three datasets have changed this picture, and all are included in Fig 1: ${ }^{4}$ MASSIV [15] derived essentially flat gradients in a large number of disks (both isolated and interacting) at $z \sim 1$. Conversely, Yuan et al. [24] and Jones et al. [8], using reconstructed source plane images of gravitationally-lensed disks at $1.5<z<2.5$, found that in three out of their four systems, the inferred oxygen gradients were very steep $(-0.15 \rightarrow-0.3 \mathrm{dex} / \mathrm{kpc})$; these four are also included in Fig 1 (crosses and inverted triangle). These data remain, to date, the only ones in the literature against which our simulations can be compared.

Complicating the picture further, one could try and make use of planetary nebulae sub-types within the Milky Way; as planetaries arise from stars ranging from $\sim 1 \mathrm{M}_{\odot}$ to $\sim 8 \mathrm{M}_{\odot}$, they provide something of a temporal probe of abundance gradients, albeit not measured in situ, but instead measured today at $z=0$, after experiencing several Gyrs or more of potential kinematic heating (both random 'blurring' and systematic radial 'migration'). We have shown in Fig 1 the extant attempts to infer the temporal evolution of the Milky Way's gradient using such planetaries; the work of Maciel et al. (diamonds) and Stanghellini \& Haywood (circles) reflects the state of the field. Unfortunately, there remains a significant discrepancy between the claimed behaviour; ${ }^{5}$ the Maciel et al. [10] analysis results in an inferred gradient for the Milky Way which was steep at early times and flattened to today's value $(-0.04 \mathrm{dex} / \mathrm{kpc}$ : Rupke et al. [16]). Conversely, the Stanghellini \& Haywood [20] work leads to an inferred gradient at early times which is somewhat flatter than today's value (hence, a steepening with time).

Finally, it is worth reminding the reader as to the predicted temporal evolution of the gradients from classical 'analytical' chemical evolution models for the Milky Way [6,11, 18]. As reported in Pilkington et al. [12], the models of Chiappini et al. [6] and Molla \& Diaz [11] show behaviour which is indistinguishable from that seen in the stronger feedback MaGICC-g1536 and MaGICCg15784 models of Fig 1, here. The model of Schönrich \& Binney [18] is similar, in the sense of showing very little temporal evolution, albeit it remains somewhat steep at all times.

In light of the success of the MaGICC formulation for feedback [2-4,13,22,23], it is tempting (for us, of course) to trumpet the consistency between classical models of chemical evolution, MaGICC-g1536, and MaGICC-g15784, with the empirical data of Queyrel et al. [15] and the planetary nebula work of Stanghellini \& Haywood [20]. Having said that, we have no a priori reason to doubt the gradients inferred from lensed disks at high-redshift $[8,24]$, nor to dismiss the planetary nebulae work of Maciel et al. [10]. Future observational campaigns, and detailed intercomparisons of the disparate planetary nebulae samples, will surely provide definitive and pivotal conclusions as to whether the somewhat flat and temporally invariant gradients predicted with the MaGICC feedback formulation stand the test of time, or whether the situation is more

\footnotetext{
${ }^{4}$ Our Fig 1 here is a preferred update to Fig 5 of [12].

${ }^{5}$ Very uncertain distance determinations and nebular emission approaches, presumably cloud the issue, but this is beyond the scope of our abilities to disentangle.
} 
complicated (or at least varied) than we envision. Such observations are a unique and, until now, missing constraint/boundary condition on models of galaxy evolution.

A characteristic of the model of Schönrich \& Binney [18], relative to several models of galactic chemical evolution $[6,11]$, is the inclusion of radial gas flows. Such flows are also a natural outcome of our MaGICC feedback scheme [1]. While challenging to infer the signal of $\sim 1-2 \mathrm{~km} / \mathrm{s}$ flows, when superimposed upon a (say) $\sigma_{r} \sim 30 \mathrm{~km} / \mathrm{s}$ velocity dispersion profile (both within the simulations and in nature [7]), we have attempted to do so. Formally, for MUGS-g1536 (at $z=0$ ), we find inward radial (cold) gas flows of $\sim 3 \mathrm{~km} / \mathrm{s}(\sim 1 \mathrm{~km} / \mathrm{s})$ within a $\pm 2 \mathrm{kpc}$ thick annulus at $13<r<17 \mathrm{kpc}$ ( $7<r<8 \mathrm{kpc}$ ); for MaGICC-g1536, the radial flows (again, at $z=0$ ) are larger: $\sim 12 \mathrm{~km} / \mathrm{s}(\sim 8 \mathrm{~km} / \mathrm{s})$ at the same galactocentric radii. A more detailed analysis of the temporal evolution of the flows will be required to disentangle the relative roles, within MaGICC, of re-cycling of the ISM over increasingly large galactic scales and the increasingly more substantial radial gas flows.

\section{Age-Metallicity Relations}

In Fig 2, for MaGICC-g1536 (left column) and MUGS-g1536 (right column), we show their inferred local 'solar' neighbourhoods' $\left(3<\mathrm{r}_{\mathrm{d}}<3.5\right)$ stellar metallicity distribution function (MDF: top row), age-metallicity relation (AMR: second row), $[\mathrm{O} / \mathrm{Fe}]-[\mathrm{Fe} / \mathrm{H}]$ distribution (third row), and $[\mathrm{O} / \mathrm{Fe}]$ distribution function (bottom row). While the impact of the enhanced feedback associated with MaGICC is readily apparent in the AMR and $[\mathrm{O} / \mathrm{Fe}]-[\mathrm{Fe} / \mathrm{H}]$ planes, the effect is more subtle in the MDF and $[\mathrm{O} / \mathrm{Fe}]$ distribution function. The remarkably tight (effectively, temporally invariant scatter) and correlated AMRs in the (analogous) simulated solar neighbourhood, for this $\mathrm{L}^{*}$ realisation, is similar to that encountered in our earlier work on dwarf disks [14]. At a given [Fe/H], within the solar neighbourhood, two (roughly) parallel loci in $[\mathrm{O} / \mathrm{Fe}]$ co-exist (for $[\mathrm{Fe} / \mathrm{H}]>-1$ ), much as seen in the Milky Way. An earlier detailed examination of the origin of these offset sequences (in a dwarf disk realisation) demonstrated significant parallels with said empirical sequences (drawing links with 'thin' and 'thick' disks, and highlighting the role of radial migration [2]); such behaviour, including offset loci in $[\mathrm{O} / \mathrm{Fe}]$ for $[\mathrm{Fe} / \mathrm{H}]>-1$ is reflected in the $\mathrm{L}^{*}$ realisations described here (see the bottom row of Fig 2 , where the bimodal $[\mathrm{O} / \mathrm{Fe}]$ distributions associated with $[\mathrm{Fe} / \mathrm{H}]>-1$ stars in the simulated solar neighbourhoods is apparent.

\section{References}

[1] Brook, C.B., et al., 2011, MNRAS, 415, 1051

[2] Brook, C.B., Stinson, G.S., Gibson, B.K., et al., 2012c, MNRAS, 426, 690

[3] Brook, C.B., Stinson, G., Gibson, B.K., et al., 2012b, MNRAS, 424, 1275

[4] Brook, C.B., Stinson, G., Gibson, B.K., et al. 2012a, MNRAS, 419, 771

[5] Chabrier, G., 2001, ApJ, 554, 1274

[6] Chiappini, C., Matteucci, F. \& Romano, D., 2001, ApJ, 554, 1044

[7] Dame, T.M., 1993, AIP Conf. Proc., 278, 267

[8] Jones, T., et al., 2012, ApJ, submitted [astro-ph / 1207 . 4489]

[9] Kroupa, P., Tout, C.A. \& Gilmore, G., 1993, MNRAS, 262, 545

[10] Maciel, W.J., Costa, R.D.D. \& Uchida, M.M.M., 2003, A\&A, 397, 667

[11] Mollá, M. \& Díaz, A.I., 2005, MNRAS, 358, 521 

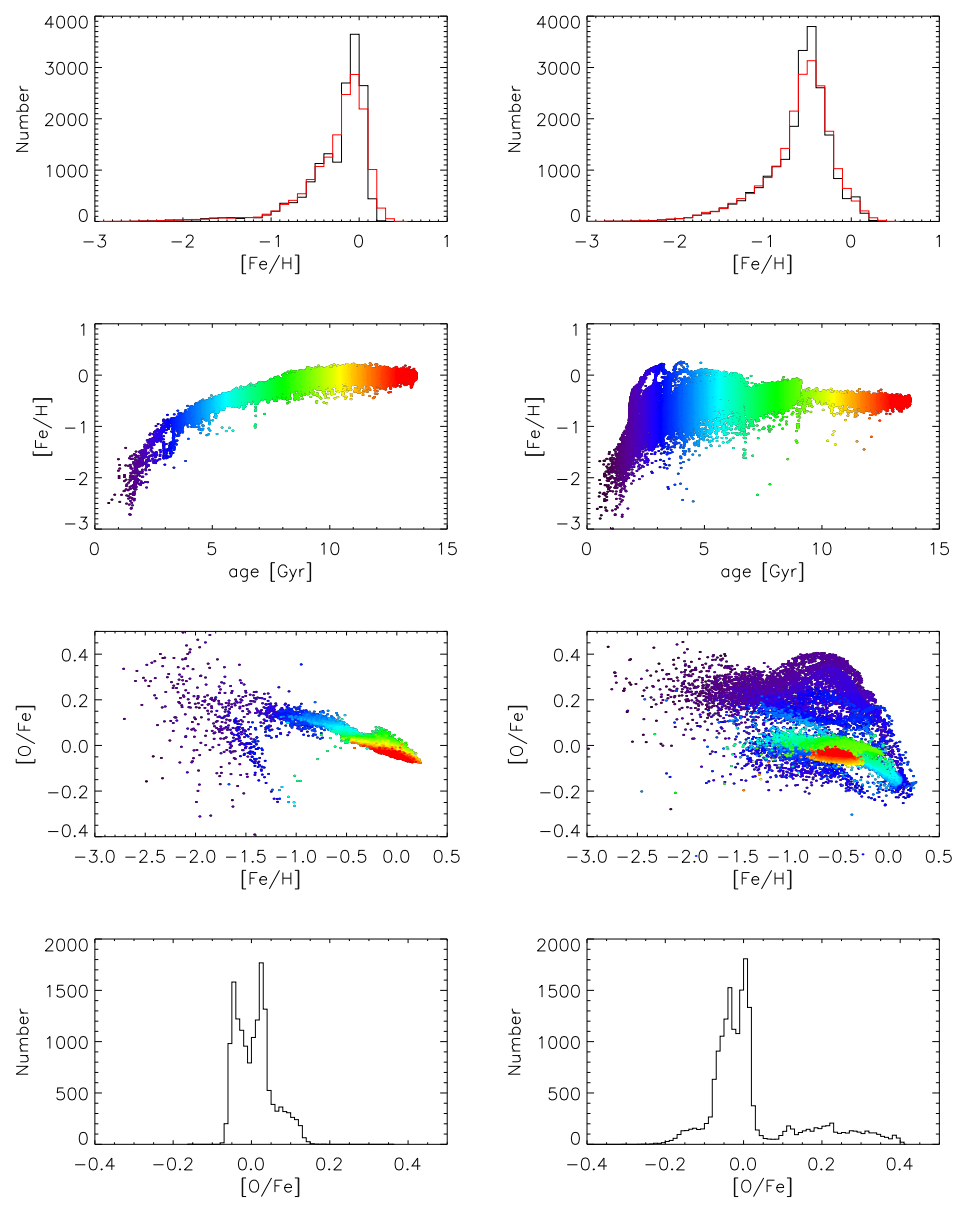

Figure 2: Predicted metallicity distribution functions (top row), age-metallicity relations (second row), $[\mathrm{O} / \mathrm{Fe}]-[\mathrm{Fe} / \mathrm{H}]$ relations (third), and $[\mathrm{O} / \mathrm{Fe}]$ distributions (bottom row), for the MaGICC (left column: [3]) and MUGS (right column: [21]) realisations of simulation g1536.

[12] Pilkington, K., Few, C.G., Gibson, B.K., et al., 2012, A\&A, 540, A56

[13] Pilkington, K. \& Gibson, B.K., 2012, ASP Conf. Ser. Vol. 458, p.241

[14] Pilkington, K., Gibson, B.K., et al., 2012, MNRAS, 425, 969

[15] Queyrel, J., et al., 2012, A\&A, 539, A93

[16] Rupke, D.S.N., Kewley, L.J. \& Barnes, J.E., 2010, ApJ, 710, L156

[17] Sánchez-Blázquez, P., Courty, S., Gibson, B.K., et al., 2009, MNRAS, 398, 591

[18] Schönrich, R. \& Binney, J., 2009, MNRAS, 396, 203 (SB09)

[19] Spitoni, E. \& Matteucci, F., 2011, A\&A, 531, A72

[20] Stanghellini, L. \& Haywood, M., 2010, ApJ, 714, 1096

[21] Stinson, G.S., Bailin, J., et al., 2010, MNRAS, 408, 812

[22] Stinson, G.S., Brook, C., Prochaska, J.X., et al., 2012, MNRAS, 425, 1270

[23] Stinson, G.S., Brook, C., Maccio, A.V., et al., 2012, MNRAS, in press

[24] Yuan, T.-T., et al., 2011, ApJ, 732, L14 